\title{
Phytogenic Synthesis of Ag Bionano-Antibiotics Against ESKAPE Drug Resistant Communities in Krasnoyarsk, Siberia
}

\author{
Baker Syed, Svetlana V. Prudnikova, Olga V. Perianova, Sergey M. Zharkov, Andrey Kuzmin, Raghuraj \\ Singh Chouhan, Nadezhda K. Potkina, Olga Y. Khohlova, Tatiana I. Lobova, Manoj Singh
}

1. Department of Microbiology Krasnoyarsk State Medical University named after Prof. VF. Voino-Yasenetskiy Krasnoyarsk Russian Federation

2. Siberian Federal University Krasnoyarsk Russian Federation

3. School of Fundamental Biology and Biotechnology Siberian Federal University Krasnoyarsk Russian Federation

4. Federal Research Center KSC SB RAS Kirensky Institute of Physics Krasnoyarsk Russian Federation

5. School of Petroleum and Natural Gas Engineering Siberian Federal University Krasnoyarsk Russian Federation

6. Department of Environmental Sciences Jožef Stefan Institute Ljubljana Slovenia

7. Department of Biotechnology, M. M. Engineering College Maharishi Markandeshwar University (MMU) Ambala India

Corresponding author:

Syed Baker*

Department of Microbiology Krasnoyarsk State Medical University named after Prof. VF. Voino-Yasenetskiy Krasnoyarsk Russian Federation

Siberian Federal University, Svobodnyy pr., 79,

Krasnoyarsk 660041, Siberia, Russian Federation

\begin{abstract}
The present study reports synthesis of silver nano-antibiotics from aqueous extract of $\mathrm{H}$. dissectum. The nano-antibiotics were characterized using UV-visible spectra which displayed maximum absorbance at $430 \mathrm{~nm}$. The role of phytocomponents as reducing agent and stabilization of silver nano-antibiotics was depicted with Fouriertransform infrared spectroscopy which revealed presence of hydroxyl and carboxyl groups associated with silver nano-antibiotics. The crystalline nature was studied with X-ray diffraction which showed Bragg's intensities at $2 \theta$ angle which denoted (101), (111), (200), (220), (311) planes. The morphological characteristics were studied using Transmission Electron Microscopy to reveal the size ranging from 5 to $50 \mathrm{~nm}$. The bactericidal activity of nanoantibiotics was studied via well diffusion and minimal inhibitory concentration assay against members of drugresistant communities bearing resistant to more than ten standard antibiotics. Nano-antibiotics displayed highest activity against Ps. aeruginosa strain 55 and Methicillin-resistant Staphylococcus aureus with $16 \mathrm{~mm}$ zone of inhibition followed by Kl. pneumoniae strain 104 and E. coli strain 55. Moderate activity was observed against Ac. baumannii strains with $12 \mathrm{~mm}$ zone of inhibition. The obtained results envision the role of plant mediated nanoantibiotics as one of the best suited alternatives towards combating drug resistant pathogens.
\end{abstract}

\section{Introduction}

The emergence of antibiotic resistant era has resulted owing to the expansion of multi drug resistant pathogens (Lobanovska and Pilla 2017). The proliferation of antimicrobial resistance has tempered the global economy by influencing health and biomedical sectors (Sharma et al. 2018). According to the latest survey, fatality ratio owing to drug resistant pathogens is increasing especially in hospital acquired infections (Kraker et al. 2016; Khan et al. 2017). The resistance is a natural phenomena wherein the pathogens are reported to undergo rapid genetic variation (King et al. 2016). Some of the major causes for drug resistance includes inappropriate prescription of standard antibiotics, poor sanitary conditions and less scientific knowledge (Raspopovic et al. 2016). The recent studies envisions the impact of hospital acquired infections with at least $70 \%$ of infections are caused by bacteria which are resistant to one or more antibiotics (Spijk et al., 2017). Especially ESKAPE members which includes Enterococcus faecium, Staphylococcus aureus, Klebsiella pneumoniae, Acinetobacter baumannii, Pseudomonas aeruginosa and Enterobacter species (Pendleton et al. 2013; Santajit and Indrawattana 2016). These pathogens are reported to be enduring the global drug resistance that prevalent in both developed and under developed countries. Some of the microbial infections caused by drug resistant are urinary tract infections, pneumonia, tuberculosis, nosocomial infections, gonorrhoea, HIV, malaria and influenza (Pendleton et al. 2013; Baker et al. 2015). Hence there is great demand to develop novel antimicrobial agents which can combat the drug resistant pathogens. According to WHO, the development of new and safe antibiotics which can act efficiently against targeted pathogens with minimal limitations is recognized as one of the top priority research of 21st century (WHO 2017). Based on these conditions, design of rational strategies by implementing scientific advances coupled with traditional 
knowledge has gained momentum. One such area accelerating in current scenario is to develop novel and safe nanoantibiotics (Syed et al. 2016). Nano-antibiotics are the class of new antimicrobial agents miniaturized at nano-scale (Huh and Kwon 2011). The recent advances of nanotechnological research has up lifted the technological aspects to produce the multi-applicative nanomaterials (Nobile and Nobile 2017). The physico-chemical properties of the nanomaterials makes them one of the most inspiring materials in comparison to their bulk counterparts (Djokic et al. 2013). The size dependent properties confers the quantum confinements and dominance of surface plasmon resonance (Gatoo et al., 2014). These unique properties portrait nanomaterials as particles of the century. The untold application of nanomaterials have already demonstrated significant progress in the field of biosensing, drug delivery, packaging, semiconductors, fuel cells, nanoagroparticles, food preservatives and nano-antibiotics (Syed et al. 2016). Scientific studies have demonstrated the effectiveness of nano-antibiotics which act on pathogens by binding to the cell wall followed by depolarizing the contents of the cell wall membrane which results in the loss of cellular contents (Syed et al. 2017). Studies also highlight that nano-antibiotics interact with the vital components of pathogens and influence the metabolic and genetic pathways, which leads to inactivation of enzymes and prevent replication (Baker and Satish, 2012). In spite of potent activity of nano-antibiotics, there has been serious concern over the mode of development. Most of the methods employed are bound with implications. Hence scientific communities have shifted their interest to produce nano-antibiotics using biogenic sources like plants and microorganisms. The plant mediated nano-antibiotics are synthesized from plants or their products which offers facile route in contrast to conventional methods which are often associated with constrains (Kavitha et al. 2013). The potential of plants to synthesized multi-functional nanomaterials can be attributed towards their potential for phytoremediation of various elements and breakdown of nutrients (Baker et al. 2013). However, studies also highlights plant mediated nanoparticles offers certain advantages over other biogenic route to synthesize nanoparticles (Iravani et al., 2014). For instance, plant mediated synthesis is one pot-synthesis with fewer requirements (Ahmed et al. 2016). The diverse classes of phyto-constituents such as terpenoids, alkaloids, flavonoids, vitamins, polyphenols and phenolic acids are reported to interact with the metal salts and mediate the reduction process (Kavitha et al. 2013). These phyto-components also acts as stabilizing agents which influences the applicative properties of nanomaterials (Baker et al. 2013). Based on these facts, in the present investigation Heracleum dissectum was selected as source to synthesize efficient nano-antibiotics which was evaluated against multi drug resistant pathogens which were isolated from patients suffering from myriad microbial infections. The cultures were maintained at Krasnoyarsk State medical University under strict aseptic conditions. The selection of plant $H$. dissectum was carried out based on the ethno-pharmacological documentation which reports its usage from centuries (Zhang et al. 2017a). The plant H.dissectum belongs to Apiaceae family which is widely distributed as perennial herb across the eatern russia and northern part of china and used as delicious food (Zhang et al. 2017a). The traditional records report the usage of this plants by local Siberian to treat diabetics, curing rheumatoid, and pain killer (Gao et al. 2014). To best of our knowledge, H. dissectum is less explored plant species which is reported to bear significant therapeutic index. Hence in the present study, H. dissectum became the subject of interest to synthesize silver nano-antibiotics.

\section{Materials and Methods}

\subsection{Plant processing}

The plant $H$. dissectum (Stem and leaves) was collected near the forest area collected around Siberian federal University, Krasnoyarsk region, Siberia, Russia. Plant was throughly washed under tap water to remove the soil debris followed by rinsing with double distilled water. The healthy plant materials were chopped into small segments and $20 \mathrm{~g}$ of finely cut materials was added to one-liter beaker containing $500 \mathrm{ml}$ of sterile distilled water (Syed et al., 2017). The mixture was boiled for 30 minutes to obtained aqueous extract which was filtered and stored stored at $4^{\circ} \mathrm{C}$ until further use.

\subsection{Synthesis of nano-antibiotics}

The aqueous extract was treated with $1 \mathrm{mM}$ silver nitrate and monitored. The conversion of $\mathrm{Ag}+$ to $\mathrm{Ag}^{\mathrm{o}}$ was initially confirmed with a change in the color of the reaction mixture and further confirmation was achieved with the UV-Visible spectrophotometer. Further, the synthesis was studied under the influence of different parameters with temperature ranging from 20 to $100{ }^{\circ} \mathrm{C}, \mathrm{pH}$ from acidic to alkaline and different concentration of silver nitrate was varied from 0.5 to $2 \mathrm{mM}$. Samples were drawn periodically to monitor the synthesis of nanoantibiotics and analyzed using UV-Visible spectrophotometer.

\subsection{Characterization of nano-antibiotics}

The synthesized $\mathrm{n}$ nano-antibiotics were characterization with sophisticated hyphenated techniques. The morphological structure was with high-resolution transmission electron microscope (HRTEM) JEOL JEM-2100 operating at an acceleration voltage of $200 \mathrm{kV}$. The HRTEM with an energy-dispersive spectrometer Oxford Inca xsight (Mikhlin et al., 2014). The possible role of the aqueous extract as reducing agent was studied using FTIR 
spectroscopy. The crystalline nature was studied using X-ray diffractometer instrument operating at a voltage of 30 $\mathrm{kV}$.

\subsection{Multi-drug resistant pathogens}

The selected strains are reported to be multi-drug resistant strains bearing resistant mechanism to nearly 10 different antibiotics. The test pathogens are Acinetobacter baumannii strain 210, Acinetobacter baumannii strain 211, Pseudomonas aeruginosa strain 55, Pseudomonas aeruginosa strain 40, Klebsiella pneumoniae strain 104, Methicillin-resistant Staphylococcus aureus, Escherichia coli strain 55. All the test pathogens were handled with prime care and preserved according to standard guidelines and maintained at culture collection center of Krasnoyarsk Medical University.

\subsection{Preparation of test bacterial suspension}

The preparation of inoculum was carried as per the Clinical and Laboratory Standards Institute (CLSI) guidelines. The test inoculum was prepared according to the protocol described by Teh et al., 2017 with slight modification. In brief, the actively growing test strains were inoculated into $10 \mathrm{ml}$ sterile Mueller Hinton broth (MHB) and incubated overnight at $37^{\circ} \mathrm{C}$. The overnight test bacterial suspensions were optically monitored and the absorbance was adjusted to $0.5 \mathrm{McF}$ arland Standard with sterile Mueller Hinton broth under aseptic conditions.

\subsection{Antimicrobial activity of nano-antibiotics}

The synthesized nano-antibiotics were centrifuged at 15,000 rpm for 20 minutes. The obtained pellet was washed thrice with double distilled water and $5 \mathrm{mg} / \mathrm{ml}$ concentration was evaluated for antimicrobial activity via well diffusion assay. In brief pre-warmed MHA (Mueller-Hinton agar) plates were seeded with test bacterial suspension $\left(1.5 \times 10^{6} \mathrm{CFU} / \mathrm{ml}\right)$ and swabbed uniformly, later by using sterile cork borer agar was punched to obtained wells and $100 \mu 1$ nano-antibiotics were added into each well and incubated at $37^{\circ} \mathrm{C}$ for 24 hours. After incubation, the zone of inhibition was measured and interpreted with different antibiotics.

\subsection{Minimal inhibitory concentration}

The minimal inhibitory concentration (MIC) was determined according to protocol described by Syed et al., 2017b. In brief, the plates were prepared under aseptic conditions and volume of $100 \mu \mathrm{L}$ of test material (nanoantibiotics $1 \mathrm{mg} / \mathrm{ml}$ ). The test material was pipette it out in the first row followed by addition of $50 \mu \mathrm{L}$ of nutrient broth to all other wells. Further, serial dilutions were performed using a multichannel pipette and $10 \mu \mathrm{L}$ of resazurin as growth indicator was seeded to each well. The final volume of the broth was adjusted with the addition of $30 \mu \mathrm{L}$ isosensitised broth to each well ensuring the final volume of the nutrient broth. Finally, $10 \mu \mathrm{L}$ of bacterial suspension $\left(1.5 \times 10^{6} \mathrm{CFU} / \mathrm{ml}\right)$ was added to each well. The plate was incubated at $37^{\circ} \mathrm{C}$ for 18 to 24 hours. The color change was then assessed visually from purple to pink or colorless. The lowest concentration at which color change occurred was taken as the MIC value (Sarker et al., 2007).

\section{Results and Discussion}

The aqueous extract of $\mathrm{H}$. dissectum forms one of the facile routes to synthesize silver nano-antibiotics. In the present investigation, the synthesis was completed within $20 \mathrm{~min}$ of reaction under the influence of optimized parameters. The synthesis was monitored with change in the color of reaction mixture. The color intensity increased and synthesis was confirmed with spectroscopic analysis using UV-visible spectroscopy which displayed the broad absorption between 200 and $800 \mathrm{~nm}$ with maximum absorption occurring at $430 \mathrm{~nm}$ (Fig. 1a). The UV-visible spectroscopy forms one of the ideal and sensitive tools to confirm the formation of silver nanomaterials. The increased in the intense and absorption is due to the surface plasmon resonance which describes the collective excitation of electrons. The excitation of electrons with change in energy levels reflects the reduction of metal ions to form nano-sized materials. The obtained results coincide with findings of Anandalakshmi et al. [2015] which indicate the presence of phyto-components in aqueous extract mediating synthesis.

The morphological characteristics was determined using TEM which displayed polydispersity with size ranging from 5 to $50 \mathrm{~nm}$ which was also confirmed with dynamic light scattering as shown in the Fig. 1b. The size and shape of nano-antibiotics can be modulated to achieve definite morphological characteristics based on the parameters such as concentration of plant extract and metal salt composition. The bio-molecular interaction between the phyto-components and silver nano-antibiotics was predicted with FTIR analysis which displayed vibrational stretches (Fig. 2a) in the IR range which corresponds to functional groups (Table 1). The influence of phytocomponents in stabilization of nanomaterials has been well documented. The diverse classes of phyto-components like polyphenols, tannic acids, vitamins, terpenoids, alkaloids, flavonoids and saponins are reported to act as reducing agents to synthesize nanomaterials. These phyto-components are well described to stabilize the nanomaterials unlike the other conventional process wherein additional stabilizing agents are supplemented during the synthesis process. The phyto-components are also reported to adhere to nanomaterials which play vital role towards achieving specific activity. The plant mediated nanomaterials is said to have better advantageous especially 
in conferring biological activities like antimicrobial. The crystalline nature of nano-antibiotics was depicted by XRD analysis which displayed (101), (111), (200), (220), (311) Bragg's peaks at 2 theta angle conferring the face centric cube of crystalline silver (Fig. 2b). The obtained result is in accordance with earlier findings of Patra et al. [2016].

There are different factors which influences the maximum synthesis process. The synthesis was minimal at lower temperatures such as 20,30 and $40{ }^{\circ} \mathrm{C}$. Further, synthesis was moderate between 50 and $70{ }^{\circ} \mathrm{C}$ which indicated elevated temperature favoring the synthesis. The synthesis was maximum synthesis at $90{ }^{\circ} \mathrm{C}$ which was monitored by measuring the absorbance at $430 \mathrm{~nm}$ (Fig. 3a). Influence of higher temperature can be related to reduction kinetic and aggregation of atoms to form nuclei followed by ripening and crystal growth to achieve particle. Earlier studies demonstrated that during plant mediated synthesis, size of the nanoparticles decreases with the increase in the temperature of the reaction mixture.

Apart from temperature, influence of $\mathrm{pH}$ was studied to determine its effect on rapid and maximum synthesis of nano-antibiotics. The $\mathrm{pH}$ was varied in the range of $\mathrm{pH} 5-10$ and rate of synthesis was monitored. At acidic $\mathrm{pH}$, the synthesis rate was much lower than the alkaline $\mathrm{pH}$ as shown in the Fig. 3b. The scientific studies demonstrated that $\mathrm{pH}$ play important role in the stability of nanomaterials. In the present investigation, increment in the rate of synthesis was observed at alkaline $\mathrm{pH}$. The concentration of metal salts was also determined between 0.5 and $2 \mathrm{mM}$, the rate of synthesis was maximum at $1 \mathrm{mM}$ concentration in comparison with other concentration (Fig. $3 c$ ). Large number of scientific studies have reported that $1 \mathrm{mM}$ concentration is ideal for rapid synthesis with sufficient amount of $\mathrm{Ag}$ precursor available for production process. The influence of different parameters to mediate, stabilize and attenuate specific properties is well demonstrated

The bactericidal properties of nano-antibiotics was tested against pathogens which were recorded to have resistant for ampicillin, cefoperazone, cefepime, chloramphenicol, imipenem, meropenem, gentamicin, tetracycline, tobramycin and vancomycin antibiotics (Table 2). The activity was determined via well diffusion assay and measured as zone of inhibition across the well and statistically analyzed (Fig. 4a). Among all the test pathogens, highest activity was observed against Ps. aeruginosa strain 215 and MRSA with $16 \mathrm{~mm}$ zone of inhibition followed by Kl. pneumoniae strain 104 and E. coli strain 55. Moderate activity was observed against Ac. baumannii strains with $12 \mathrm{~mm}$ zone of inhibition. The activity was in accordance with broth dilution assay which displayed decrease in the optical density of test pathogens in presence of nano-antibiotics (Fig. 4b). The activity was further confirmed with minimal inhibitory concentration assay which displayed minimal activity obtaining between the concentration $0.625 \mu \mathrm{g} / \mathrm{ml}$ to $1.25 \mu \mathrm{g} / \mathrm{ml}$ (Table 3). Perusal of scientific literatures on $\mathrm{H}$. dissectum describes scanty reports with few scientific studies working on this edible plant. Recently, a new classes of phenylpropanoids, glycosides, coumarins and neolignans were isolated from the roots of $\mathrm{H}$. dissectum which were reported to possess cytotoxic activity. Much more studies are essential and required to reveal the untapped phyto-components from H. dissectum. In the present investigation, the antibacterial activity of plant mediated nanoparticles was compared with both chemically synthesized nanoparticles and the aqueous extract of $\mathrm{H}$. dissectum. Interestingly, chemically synthesized nanoparticles had no effect on drug resistant test pathogens. The concentrated aqueous extract showed moderate activity which indicated the presence of active compounds from the plant which forms the capping across the particle to add bactericidal activity. The obtained results are promising enough for future investigation to elucidate the exact mechanism responsible synthesis and role of phyto-components in bactericidal activity. Overall, the obtained results attributes towards growing knowledge of developing new and safe antimicrobial agents. Even though considerable investigation are being carried out on different classes of nanomaterials are conducted, scanty reports are available on multi-drug resistant pathogenic microorganisms which are clinically significant and belonging to ESKAPE group. The obtained results coincides with the findings of Thiagarajan and Sangappa [2015] which reports the activity of silver oxide nanoparticles against MRSA strain which was clinically isolated. In the present investigation, activity was observed against both Gram -ve and Gram +ve pathogenic bacteria which indicates the broad spectrum activity of synthesized nano-antibiotics.

Large number of scientific studies illustrates the antibacterial activity of silver nanomaterials against pathogenic microorganisms. The activity of nanomaterials depends on various parameters for instance, the size of the nanomaterials smaller the size, greater will be the activity. The surface area of the nanomaterials higher the surface area, significant will be the activity of nanomaterials against targeted pathogen. Initially, the Ag+ released binds to the membrane proteins and enzymes to form bond which results in the suppression and deactivation of the protein. Studies also confer the obstruction of respiratory enzymes by silver nanomaterials. Furthermore, the source of synthesizing nanomaterials also contributes to attenuate antimicrobial activity against pathogens especially from biogenic source of synthesis of nanomaterials. In the present investigation, polydispersity of nano-antibiotics play important role to suppress the growth of the test pathogens. The selected plant species to synthesize nano antibiotics is reported to have therapeutic index as per the traditional records which can also participate in antibacterial activity. The nano-antibiotics synthesized in the present study displayed significant activity against Gram +ve and Gram -ve 
test pathogens which are isolated from clinical samples of patients suffering from myriad infections. Further study in this area will be highly valuable to elucidate exact mechanism for antibacterial activity. The interaction of nanomaterials most of the pathogenic microorganisms have modulated the growth regulations process which can be used as template based probe to identify the degree of resistance and susceptibility to design the monitoring of drug resistant pathogens.

\section{Conclusion}

The activity obtained in the present investigation is promising enough to report the synthesis of silver nanoantibiotics using H. dissectum. The silver nano antibiotics displayed bactericidal activity via well diffusion assay and minimal inhibitory concentration against members of ESKAPE pathogens which are reported to be one of the leading causes of nosocomial infections. Overall the study forms initial findings which will be further evaluated for investigation to reveal the mechanism of synthesis and activity. The study also makes an attempt to evaluate nanomaterials as one of the best suited alternatives to combat drug resistant pathogens.

\section{Acknowledgements}

Authors are thankful for facilities provided by Siberian Federal University to carry out the present study. Authors are grateful to Krasnoyarsk Medical University for providing opportunity to carry out the present study.

Table 1. FTIR peak position of all functional groups

\section{FTIR Functional group}
$3363 \mathrm{OH}$
$2918 \mathrm{CH}$
$1596 \mathrm{C}=\mathrm{O}$
$1358 \mathrm{C}-\mathrm{H}$
$1045 \mathrm{C}-\mathrm{O}$

Table 2. Data on antibiotic resistance of the strains used in the work

\section{Strain Cep Cez Cef Imp Mer Tic Gen Tob Ami Cip Chl Ctm}

\begin{tabular}{|c|c|c|c|c|c|c|c|c|c|c|}
\hline Ac. baumannii $211 \mathrm{R}$ & $\mathrm{R}$ & $\mathrm{R}$ & S & $\mathrm{R}$ & $\mathrm{R}$ & $\mathrm{R}$ & S & $\mathrm{R}$ & $\mathrm{R}$ & $\mathrm{R}$ \\
\hline Ac. baumannii $210 \mathrm{R}$ & $\mathrm{R}$ & $\mathrm{R}$ & $\mathrm{R}$ & $\mathrm{R}$ & $\mathrm{R}$ & $\mathrm{R}$ & $S$ & $\mathrm{R}$ & $\mathrm{R}$ & $\mathrm{R}$ \\
\hline Ps. aeruginosa $215 \mathrm{~S}$ & $\mathrm{~S}$ & $\mathrm{~S}$ & $\mathrm{R}$ & $\mathrm{R}$ & $\mathrm{R}$ & $\mathrm{S}$ & $\mathrm{S}$ & S & $\mathrm{S}$ & $\mathrm{R}$ \\
\hline Ps. aeruginosa 40 & $\mathrm{R}$ & $\mathrm{R}$ & $S$ & $\mathrm{R}$ & $\mathrm{R}$ & $\mathrm{R}$ & $\mathrm{R}$ & $\mathrm{P}$ & $\mathrm{R}$ & D \\
\hline Kl. pneumonia $104 \mathrm{R}$ & $\mathrm{R}$ & $\mathrm{R}$ & $S$ & $\mathrm{R}$ & $\mathrm{R}$ & $\mathrm{R}$ & $\mathrm{R}$ & $\mathrm{R}$ & $\mathrm{S}$ & $\mathrm{R}$ \\
\hline E. coli 55 & $\mathrm{R}$ & $\mathrm{R}$ & S & S & $\mathrm{R}$ & $\mathrm{R}$ & $\mathrm{R}$ & S & $\mathrm{R}$ & $\mathrm{R}$ \\
\hline
\end{tabular}

Table 3. Minimal inhibitory concentration of silver nano-antibiotics against multi-drug resistant pathogens

\section{Pathogens}

\section{Silver nano-antibiotics $(\mu \mathrm{g} / \mathrm{ml})$}

Ac. baumannii strain 2101.25

Ac. baumannii strain $211 \quad 1.25$

E. coli strain 215

0.625

Kl. pneumoniae strain 1041.25

MRSA

0.625

Ps. aeruginosa strain $40 \quad 1.25$

Ps. aeruginosa strain $55 \quad 0.625$ 

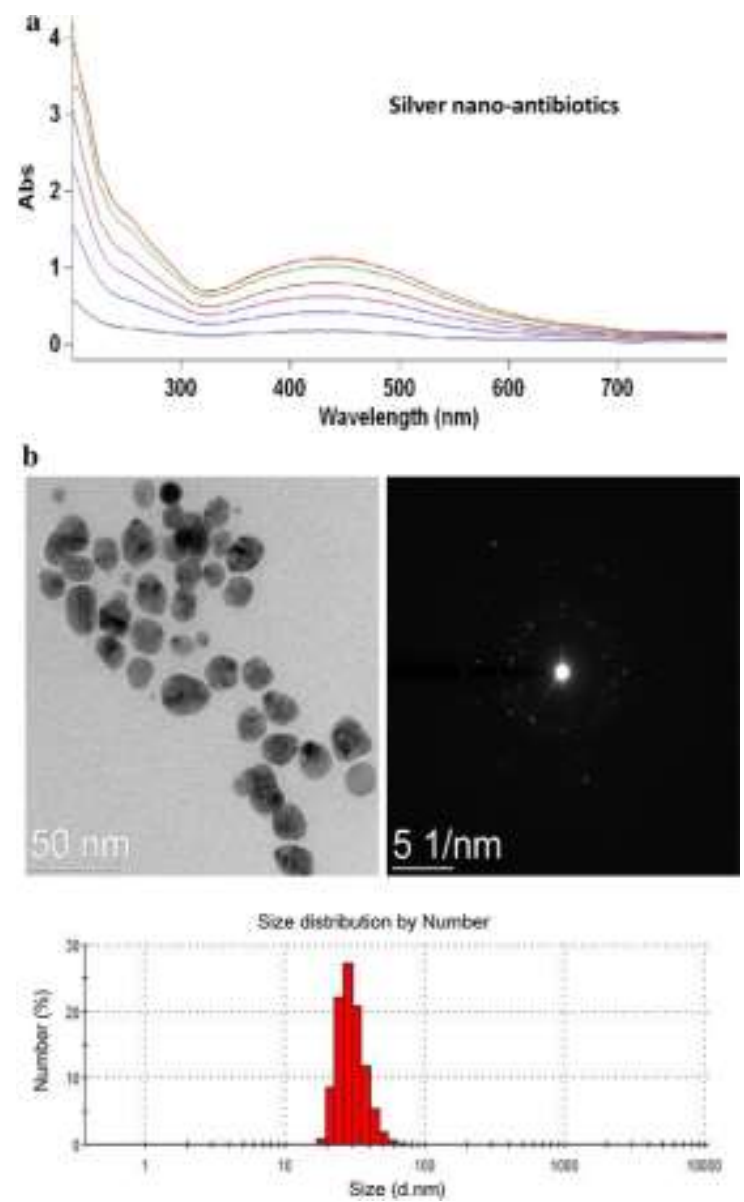

Fig. 1. a - UV-visible spectra of silver bionano-antibiotics. b - TEM analysis of silver bionano-antibiotics with DLS analysis
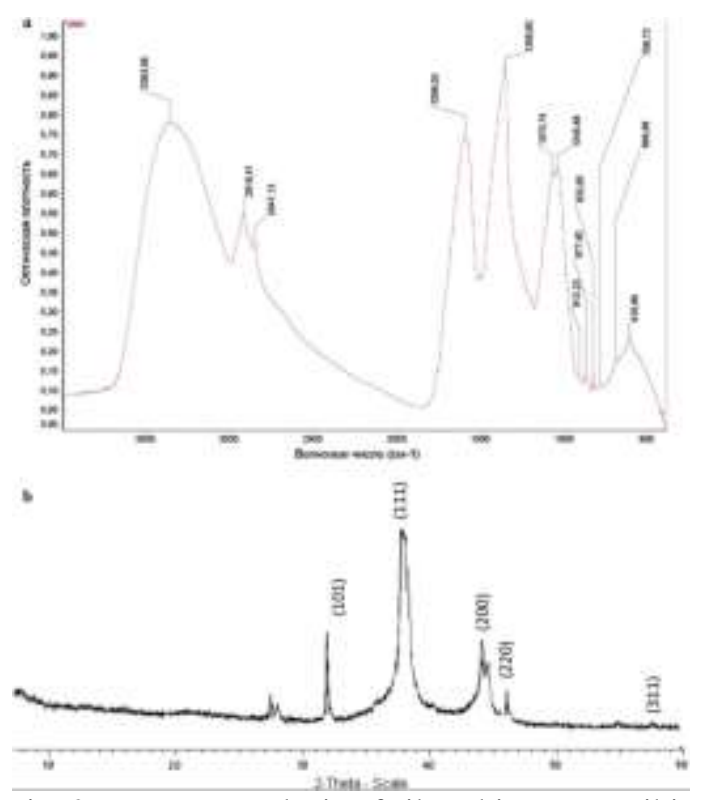

Fig. 2. a - FTIR analysis of silver bionano-antibiotics. b - XRD analysis of silver bionano-antibiotics 

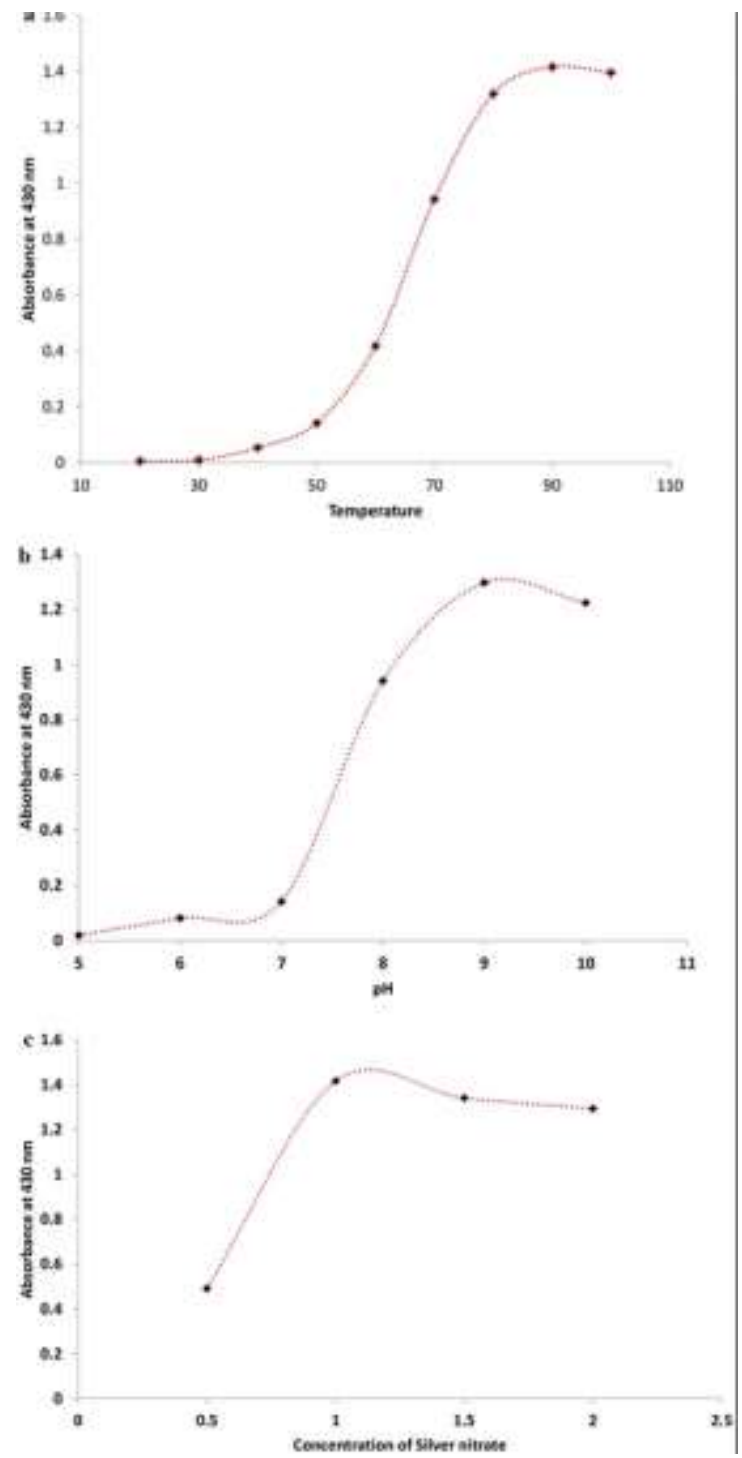

Fig. 3. a - Influence of temperature on synthesis of silver bionano-antibiotics. $\mathrm{b}$ - Influence of $\mathrm{pH}$ on synthesis of silver bionano-antibiotics. c - Influence of metal concentration on synthesis of silver bionanoantibiotics
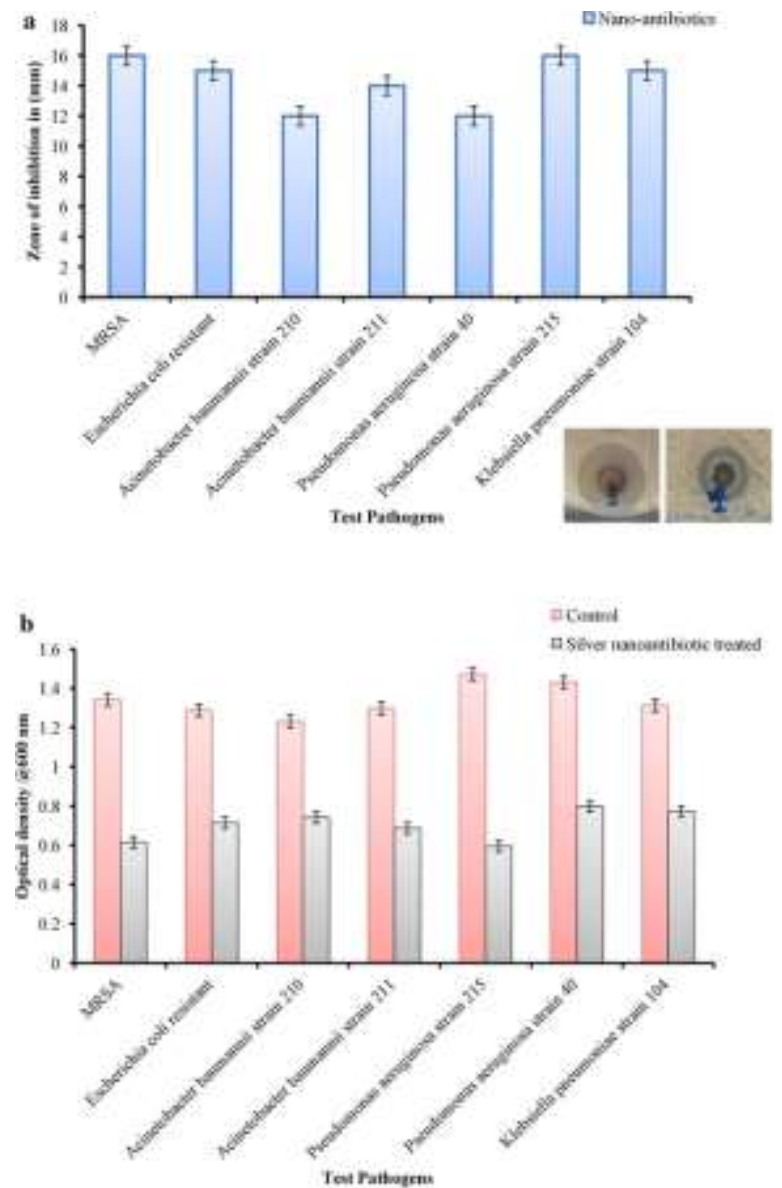

Fig. 4. a - Bactericidal activity of bionano-antibiotics against multi-drug resistant pathogens via well diffusion assay. b - Bactericidal activity of bionano-antibiotics against multi-drug resistant pathogens via broth dilution assay 